\title{
Vigilância da fluoretação das águas no Brasil: uma revisão de literatura
}

Vigilance of water fluoridation in Brazil: a review

Vigilancia de la fluoración de las aguas en Brasil: una revisión de literatura

\author{
Bárbara Faria de Sá BARBOSA ${ }^{1}$ \\ Herika de Arruda MAURICIO ${ }^{1}$ \\ Pedro Henrique SETTE-DE-SOUZA ${ }^{1}$
}

Camila Ananias de LIMA ${ }^{2}$

${ }^{1}$ Graduação em Odontologia da Universidade de Pernambuco - UPE, Campus Arcoverde, 56503-146Arcoverde-PE, Brasil

${ }^{2}$ Biotech - Soluções Inteligentes para a sua Saúde LTDA, 55014-100Caruaru - PE, Brasil

\begin{abstract}
Resumo
Introdução: Para que a qualidade da água seja mantida, a vigilância a partir do heterocontrole é fundamental. O heterocontrole garante imparcialidade ao monitoramento das águas, na medida em que a vigilância é desenvolvida por instituições não responsáveis pelo abastecimento público. Objetivo: Caracterizar o cenário brasileiro quanto à vigilância pelo heterocontrole da fluoretação das águas no período de 2002 a 2016. Material e método: Foi desenvolvida uma revisão de literatura a partir de busca com o uso dos descritores "fluoretação da água", "fluoretação", "vigilância", "vigilância epidemiológica", "qualidade da água" e "flúor". Resultados: Foram identificados 10 levantamentos epidemiológicos envolvendo essa temática. Entre esses, apenas 1 estudo verificou que 100\% das amostras apresentavam teores adequados de flúor na água distribuída para a população. Conclusão: O estudo reforça a importância do monitoramento da água consumida, em especial por meio do heterocontrole.
\end{abstract}

Descritores: Flúor; Fluoretação; Qualidade da Água; Monitoramento Epidemiológico.

\begin{abstract}
Introduction: In order for water quality to be maintained, monitoring from the heterocontrol is fundamental. The heterocontrol guarantees impartiality to the monitoring of the waters, to the extent that the surveillance is developed by institutions not responsible for the public supply. Objective: To characterize the Brazilian scenario regarding the monitoring by the heterocontrol of water fluoridation from 2002 to 2016. Material and method: A literature review was developed from the search using the descriptors "water fluoridation", "fluoridation", "surveillance", "epidemiological surveillance", "water quality" and "fluorine". Results: Ten epidemiological surveys were identified involving this theme. Among these, only 1 study verified that $100 \%$ of the samples had adequate levels of fluoride in the water distributed to the population. Conclusion: The study reinforces the importance of monitoring the water consumed, especially through the heterocontrol.

Descriptors: Fluorine; Fluoridation; Water Quality; Epidemiological Monitoring.
\end{abstract}

\section{Resumen}

Introducción: Para que la calidad del agua se mantenga, la vigilancia a partir del heterocontrole es fundamental. El heterocontrole garantiza imparcialidad al monitoreo de las aguas, en la medida en que la vigilancia es desarrollada por instituciones no responsables del abastecimiento público. Objetivo: Caracterizar el escenario brasileño en cuanto a la vigilancia por el heterocontrole de la fluoración de las aguas en el período 2002 a 2016. Material y método: Se desarrolló una revisión de literatura a partir de la búsqueda con el uso de los descriptores "fluoretación del agua", "fluoruración ", "vigilancia", "vigilancia epidemiológica", "calidad del agua" y "flúor". Resultados: Se identificaron 10 estudios epidemiológicos que involucra esta temática. Entre estos, sólo un estudio verificó que el 100\% de las muestras presentaban niveles adecuados de flúor en el agua distribuida para la población. Conclusión: El estudio refuerza la importancia del monitoreo del agua consumida, en especial por medio del heterocontrole.

Descriptores: Flúor; Fluoruración; Calidad del Agua; Monitoreo Epidemiológico.

\section{INTRODUÇÃO}

O flúor é o décimo terceiro elemento disponível em maior abundância na natureza e o mais eletronegativo da tabela periódica. Encontrado na forma de fluoreto, é liberado das rochas e transferido para as águas e $\operatorname{solos}^{1-4}$. O processo de adição de composto de flúor às águas de abastecimento público denomina-se fluoretação ${ }^{5-7}$. Apresenta-se como uma ferramenta para a prevenção da cárie, de baixo custo, grande efetividade, segura, de fácil administração e de abrangência coletiva ${ }^{8-13}$.

Quando em contato direto com os dentes na cavidade bucal, o flúor interfere no processo carioso ajudando na remineralização do elemento dentário. Ele não impede que a doença cárie ocorra, mas pode reduzir a sua progressão por meio de interações físicas e químicas. Promovendo a formação de fluorapatita, o flúor auxilia o retorno do $\mathrm{pH}$ salivar ao seu nível normal, em torno de cinco, induzindo a reposição dos minerais, e assim, impedindo a desmineralização do dente ${ }^{4,12}$.

Uma vez interrompida a ingestão de água fluoretada, os níveis de flúor na corrente sanguínea não serão mantidos, reforçando a necessidade de que a fluoretação das águas seja um processo constante para controle coletivo da cárie. A eficiência desse método preventivo foi comprovada por meio de estudos de verificação da prevalência de cárie aos 12 anos desenvolvidos em diferentes países ${ }^{12}$.

O Programa Nacional de Vigilância da Água para Consumo Humano (VIGIÁGUA) consiste em um conjunto de ações de saúde pública que objetivam garantir o atendimento das normas/padrões estabelecidos pela legislação vigente (Portaria $\mathrm{N}^{\circ}$ 518/2004) e avaliar os riscos que as águas de abastecimento podem representar à saúde humana. Cabe ao VIGIÁGUA identificar, cadastrar e inspecionar as várias formas de abastecimento para monitorar a qualidade da água, analisando e classificando quanto ao grau de risco à saúde a depender da forma de abastecimento que aquela água tem ${ }^{14}$. É estruturado com base no Sistema Único de Saúde (SUS), possuindo um papel importantíssimo na garantia de segurança na qualidade da água ${ }^{15}$.

No Brasil, o teor de $1,5 \mathrm{mg}$ F/L é adotado para o território nacional como valor máximo permitido. Esse valor foi estabelecido com base em relatórios técnicos da Organização Mundial de Saúde. Esta concentração é capaz de promover a máxima 
redução do índice CPO-D sem aumentar o índice de fluorose ${ }^{6,12,13,16}$.

Para evitar que os teores de flúor sejam inadequados na água, a vigilância sanitária é responsável pela execução de monitoramento periódico nas estações de tratamento, aplicando o que é preconizado pelo VIGIAGUA. Um segundo tipo de monitoramento é denominado de heterocontrole, o qual é realizado por instituições não envolvidas diretamente na operacionalização da fluoretação, seja por um órgão/instituição pública ou privada. Esse tipo de monitoramento independente é capaz de produzir dados confiáveis, oferecendo mais qualidade ao processo de fluoretação ${ }^{16}$.

Nesse sentido, o objetivo do presente estudo é apresentar um apanhado de revisões disponíveis na literatura científica, que caracterizem o cenário brasileiro quanto ao heterocontrole do flúor.

\section{MATERIAL E MÉTODO}

Trata-se de uma revisão de literatura de caráter exploratório, desenvolvida com o intuito de identificar material bibliográfico que permitisse traçar um panorama sobre a vigilância das águas realizada no Brasil por meio do heterocontrole. Foi desenvolvida uma busca por artigos, teses, dissertações e manuais em base de dados como a Biblioteca Virtual em Saúde (BVS), utilizando os seguintes descritores em saúde: "fluoretação da água" "fluoretação"; "vigilância"; "vigilância epidemiológica"; "qualidade da água" "flúor".

\section{RESULTADOS}

A partir da estratégia de busca desenvolvida, foram identificadas 10 referências bibliográficas abordando a vigilância da fluoretação das águas no Brasil a partir do heterocontrole. No Quadro 1, estão apresentados os resultados verificados nestes estudos.

\begin{tabular}{|c|c|c|c|}
\hline Ano & Autores & $\begin{array}{c}\text { Local do } \\
\text { estudo }\end{array}$ & Resultados \\
\hline 2002 & Pires et al. ${ }^{17}$ & $\begin{array}{l}\text { Juiz de Fora - } \\
\text { MG }\end{array}$ & $\begin{array}{l}\text { Identificaram melhora nos níveis de fluoretação, } \\
\text { apesar da sua falta de regularidade. Dos } 14 \\
\text { bairros selecionados para a pesquisa, } 6 \\
\text { apresentaram índices abaixo do teor ideal, } \\
\text { totalizando } 42,8 \% \text { dos bairros em situação } \\
\text { inadequada. }\end{array}$ \\
\hline 2003 & Maia et al. ${ }^{18}$ & Niterói - RJ & $\begin{array}{l}\text { Observaram grande incoerência entre os valores } \\
\text { obtidos na pesquisa e os relatados pela estação } \\
\text { de tratamento. } 96 \% \text { das amostras analisadas } \\
\text { estavam inadequadas. }\end{array}$ \\
\hline 2005 & Moura et al. ${ }^{19}$ & Teresina - PI & $\begin{array}{l}\text { Analisaram a concentração de flúor na água de } \\
\text { cinco bairros ao longo de } 12 \text { meses, identificando } \\
\text { que } 46,7 \% \text { das amostras apresentaram } \\
\text { concentração de flúor abaixo ou acima do padrão } \\
\text { preconizado. }\end{array}$ \\
\hline 2007 & Silva et al..$^{20}$ & $\begin{array}{c}\text { Teresina, } \\
\text { Floriano, } \\
\text { Parnaíba-PI } \\
\end{array}$ & $\begin{array}{l}\text { Verificaram que } 95,7 \% \text { das amostras coletadas } \\
\text { estavam com flúor em concentração inaceitável } \\
\text { para o controle da cárie dentária. }\end{array}$ \\
\hline 2008 & Panizzi e Peres ${ }^{21}$ & Chapecó - SC & $\begin{array}{l}\text { Perceberam que entre } 989 \text { amostras de água } \\
\text { avaliadas, } 54 \% \text {, } 68 \% \text { e } 57 \% \text { dessas estavam } \\
\text { inadequadas, a depender do critério adotado. }\end{array}$ \\
\hline 2009 & Bellé et al. ${ }^{3}$ & $\begin{array}{c}\text { Campo Grande } \\
\text { MG }\end{array}$ & 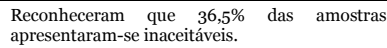 \\
\hline 2012 & Moimaz et al. ${ }^{22}$ & $\begin{array}{l}\text { Araçatuba - } \\
\text { SP }\end{array}$ & $\begin{array}{l}\text { Observaram que } 100 \% \text { das amostras coletadas } \\
\text { estavam com teores que trariam benefício } \\
\text { máximo à população. }\end{array}$ \\
\hline 2014 & Stancari et al. ${ }^{23}$ & Bauru - SP & $\begin{array}{l}\text { Para os municípios da Regional XV-Bauru/SP, } \\
\text { no período de } 2002 \text { a } 2011,8,4 \% \text { estavam acima } \\
\text { dos valores aceitáveis e } 29,4 \% \text { estavam abaixo do } \\
\text { limite desejado. }\end{array}$ \\
\hline 2015 & $\begin{array}{ll}\begin{array}{l}\text { Moimaz } \\
\text { Santos }^{24}\end{array} \quad \text { e } \\
\end{array}$ & Birigui - SP & $\begin{array}{l}\text { Notaram que } 52,38 \% \text { dos pontos de coleta } \\
\text { possúam quantidade de flúor acima dos } \\
\text { parâmetros recomendados. }\end{array}$ \\
\hline 2016 & Brito et al. ${ }^{2}$ & $\begin{array}{l}\text { Passo Fundo - } \\
\text { RS }\end{array}$ & $\begin{array}{l}\text { Em } 60 \% \text { das amostras os níveis estavam abaixo } \\
\text { do esperado. }\end{array}$ \\
\hline
\end{tabular}

Quadro 1: Vigilância da fluoretação das águas pelo heterocontrole. Brasil, 2002-2016 (Fonte: Dados da pesquisa).

\section{DISCUSSÃO}

O impacto promovido pela fluoretação das águas de consumo humano na prevenção da cárie é largamente comprovado pela literatura científica brasileira. Segundo Narvai ${ }^{25}$, no período de 1986 a 1996, 42\% da população brasileira tinha acesso à água fluoretada, possibilitando uma redução de 53\% da prevalência de cárie nas crianças de 12 anos. Comparando dois municípios baianos com e sem fluoretação das águas, Cardoso e Moraes $^{10}$ constataram a redução de $22 \%$ do índice CPO-D na população com água fluoretada. Brienza ${ }^{7}$ verificou que em Ribeirão Preto o índice CPO-D reduziu em $65 \%$ após as águas do município serem fluoretadas. Já Meirelles e Sousa ${ }^{26}$ observaram a redução de $11 \%$ do índice quando compararam escolares que faziam uso de água fluoretada com os que não tinham acesso.

Apesar disso, a população brasileira como um todo ainda não possui acesso à água de boa qualidade, com garantia de que sua concentração de flúor se encontre entre os níveis ótimos preconizados. Um dos eixos de entraves para essa garantia encontra-se no nível técnico-operacional do VIGIAGUA. Mesmo que a fundamentação do Programa apresente-se definida, diferentes questões impedem a sua adoção prática. Cadastramento e vigilância das instalações de abastecimento de água, coleta e análise de dados gerados pela vigilância, instrumentos de georreferenciamento e integração entre departamentos e setores apresentam-se deficientes, restringindo as ações do Programa basicamente a situações de surto ${ }^{27,28}$.

Por isso, torna-se necessário buscar alternativas que viabilizem o acesso a dados sobre a qualidade da água. Narvai ${ }^{25}$ define heterocontrole como "princípio segundo o qual se um bem ou serviço qualquer implica risco ou representa fator de proteção para a saúde pública então além do controle do produtor sobre o processo de produção, distribuição e consumo deve haver controle por parte das instituições do Estado". O heterocontrole da fluoretação é realizado por instituições não envolvidas diretamente em sua operacionalização, sendo imprescindível para garantir a qualidade do processo, a credibilidade das informações geradas e emitir alertas quando os níveis estiverem fora dos padrões ${ }^{16}$.

Por meio da revisão bibliográfica desenvolvida, foi possível verificar que entre as 10 referências bibliográficas identificadas, apenas uma ${ }^{29}$ mostrou-se com $100 \%$ de suas amostras atingindo níveis ótimos de concentração de flúor. Todos os demais estudos apresentaram resultados abaixo ou acima dos limites estabelecidos, confirmando que a água fornecida pode tanto estar sendo inócua para a prevenção da cárie como pode estar provocando fluorose. 
O presente estudo apresenta limitações quanto aos resultados apresentados, pelo fato de ter sido desenvolvida uma estratégia de busca exploratória das referências em bases científicas.

Mesmo assim, compreende-se que o monitoramento da fluoretação das águas realizado em diferentes localidades por meio do heterocontrole apontou para a inadequação das águas de abastecimento público na grande maioria dos casos.

\section{CONCLUSÃO}

A disponibilização de dados gerados pelo heterocontrole, combinados com dados primários, secundários, revisões bibliográficas e sistemáticas de literatura sobre a questão da fluoretação das águas de consumo humano no País produzem evidência científica essencial para o agir em saúde.

\section{REFERÊNCIAS}

1. Palmer C, Wolfe SH, American Dietetic Association. Position of the American Dental Association: the impact of fluoride health. J Am Diet Assoc;2005;105(10):1620-28.

2. Anjos GAS, Fernandes GF. Fluoretação das águas de abastecimento público no estado de Pernambuco: um resgate histórico. Odontol ClínCient. 2015;14(1):559-64.

3. Bellé BLL, Lacerda VR, Carli AD, Zafalon EJ, Pereira PZ. Análise da fluoretação da água de abastecimento público da zona urbana do município de Campo Grande (MS). Ciênc saúde coletiva. 2009;14(4):1261-66.

4. Brasil. Ministério da Saúde. Portaria $n^{\circ} 685$, de 25 de dezembro de 1975. Aprova as normas e padrões sobre a fluoretação da água dos sistemas públicos de abastecimento, destinada ao consumo humano. Brasília: Diário Oficial da União; 1975.

5. Brasil. Ministério da Saúde. Vigilância e controle da qualidade da água para o consumo humano. Secretaria de Vigilância em Saúde. Brasília - DF, 2018.

6. Agência Nacional de Saúde. Manual de fluoretação da água para consumo humano. Brasília: Funasa; 2012.

7. Brienza JA. A fluoretação das águas de abastecimento público no município de Ribeirão Preto (SP) [dissertação]. Ribeirão Preto: Universidade de São Paulo - USP; 2005.

8. Brito CS, Garbin RR, Mussi A, Rigo L. Vigilância da concentração de flúor nas águas de abastecimento público na cidade de Passo Fundo - RS. Cad Saúde Colet. 2016;24(4):452-59.

9. Burt BA, Fejerskov O. Waterfluoridation. In: Fejeskov O, Ekstrand J, Burt BA, editors. Fluoride in dentistry. Copenhagen: Munksgaard; 1996. p. 275-90.

10.Cardoso ACC, Moraes LRS. A associação entre cárie e fluorose dentária e a fluoretação das águas em dois municípios da Bahia. Rev Baiana saúde pública. 2003;27(1/2):7-18.
11.Centers for Disease Control and Prevention. Achievements in public health, 1900-1999: Fluoridation of drinking water to prevent dental caries. Morbidity and Mortality Weekly Reports October 22, 1999; 48(41);933-40.

12.Centers for Disease Control and Prevention. Engineering and administrative recommendations for water fluoridation. Morbidity and Mortality Weekly Reports 1995; 44(RR-13):1-40.

13.Centers for Disease Control and Prevention. Recommendation for using fluoride to prevent and control dental caries in the United States. Morbidity and Mortality Weekly Reports 2001; 50(n.RR-14):1-42.

14.Cesa KT. A vigilância dos teores de flúor nas águas de abastecimento público nas capitais do Brasil [mestrado]. Porto Alegre: Universidade Federal do Rio Grande do Sul - UFRS; 2007.

15. Cury JA. Uso do flúor e controle da cárie como doença. In: Baratieri $\mathrm{LN}$ et al. Odontologia restauradora. São Paulo: Santos; 2001. p.34-68.

16. Horowitz HS. The effectives of community water fluoridation in the United States. J Public Health Dent.1996;56(5 Spec No):253-58.

17.Pires LD, Macêdo JAB, Rocha HVA, Lima DC, Vaz UP, Oliveira RF. Determinação do índice de fluoreto em águas de abastecimento público na cidade de Juiz de Fora. Eng Sanit Ambient. 2002;7(1/2):21-9.

18. Maia LC, Valença AMG, Soares EL, Cury JA. Controle operacional da fluoretação da água de Niterói, Rio de Janeiro, Brasil. Cad Saúde Pública. 2003;19(1):61-7.

19. Moura MS, Silva JS, Simplicio AHM, Cury JA. Avaliação longitudinal da fluoretação da água de abastecimento público de Teresina-Piauí. Rev Odonto Ciênc. 2005; 20(48):132-36.

20.Silva JS, Val CM, Moura MS, Silva TAE, Sampaio FC. Heterocontrole da fluoretação das águas em três cidades no Piauí, Brasil. Cad Saúde Pública. 2007;23(5):1083-88.

21.Panizzi M, Peres MA. Dez anos de heterocontrole da fluoretação de águas em Chapecó, Estado de Santa Catarina, Brasil. Cad Saúde Pública. 2008;24(9):2021-31.

22.Moimaz SAS, Saliba O, Garbin CAS, Garbin AJÌ, Sumida DH, Corrêa MV, Saliba NA. Fluoretação das águas de abastecimento público no município de Araçatuba/SP. Rev Odontol Araçatuba. 2012;33(1):54-60.

23. Stancari RCA, Dias Júnior FL, Freddi FG. Avaliação do processo de fluoretação da água de abastecimento público nos municípios pertencentes ao Grupo de Vigilância Sanitária XV-Bauru, no período de 2002 a 2011. Epidemiol Serv Saúde. 2014;23(2):239-48.

24. Moimaz SAS, Santos LFP. Estudo longitudinal da fluoretação das águas em município com 
complexa rede de distribuição: dez anos de estudo. Arch Health Invest. 2015;4(5):11-16.

25.Narvai PC. Cárie dentária e flúor: uma relação do século XX. Ciênc saúde coletiva. 2000;5(2):38192.

26. Meirelles MPMR, Sousa MLR. Importância da fluoretação das águas de abastecimento público em municípios de pequeno porte na região sudoeste do estado de São Paulo. Rev Fac Odonto Porto Alegre. 2005;46(2):15-19.

27.Kozlowski FC, Pereira AC. Métodos de utilização de flúor sistêmico. In: Pereira AC, organizador. Odontologia em saúde coletiva. Porto Alegre: Artmed;2003. p. 265-74.

28. Queiroz A, Cardoso L, Silva S, Heller L, Caincross S. Programa Nacional de Vigilância da Água para Consumo Humano (Vigiagua): lacunas entre a formulação do programa e sua implantação na instância municipal. Saúde soc. 2012;21(2):465-78.

29.Nanni AS. O flúor das águas do sistema aquífero Serra Geral no Rio Grande do Sul: origem e condicionamento geológico [tese]. Porto Alegre: IGEO/UFRGS; 2008.

\section{CONFLITO DE INTERESSES}

Os autores declaram não haver conflitos de interesse.

\section{AUTOR PARA CORRESPONDENCIA}

Bárbara Faria de Sá Barbosa

barbarafb2304@gmail.com

Submetido em 25/10/2018

Aceito em 12/03/2019 